\title{
A sound strategy for gene expression
}

Imaging of gene expression in live cells or tissues has largely relied on light-based strategies detecting fluorescence or luminescence, but poor transmission of light through tissues limits the depth that can be imaged. A new study reports a synthetic biology approach to encode an ultrasound-based gene expression reporter that is applicable to mammalian cells in vitro and in vivo.

To adopt ultrasound as a robust biomolecular readout, strategies are required that convert gene expression into a change in sonic contrast. One promising approach is to take advantage of air-filled protein nanostructures encoded by some bacterial species that rely on these gas vesicles for buoyancy in water. A multigene system for producing these gas vesicles, termed acoustic reporter genes (ARGs), was previously transferred into commensal bacteria for ultrasound-based imaging in the mouse gastrointestinal tract. Now, Farhadi et al. sought to encode an equivalent system in mammalian cells.

Much of the study focused on optimizing this bacterial system for cross-kingdom expression, such as re-coding using mammalian codon optimization, determining which of the genes are key for gas vesicle generation and testing different

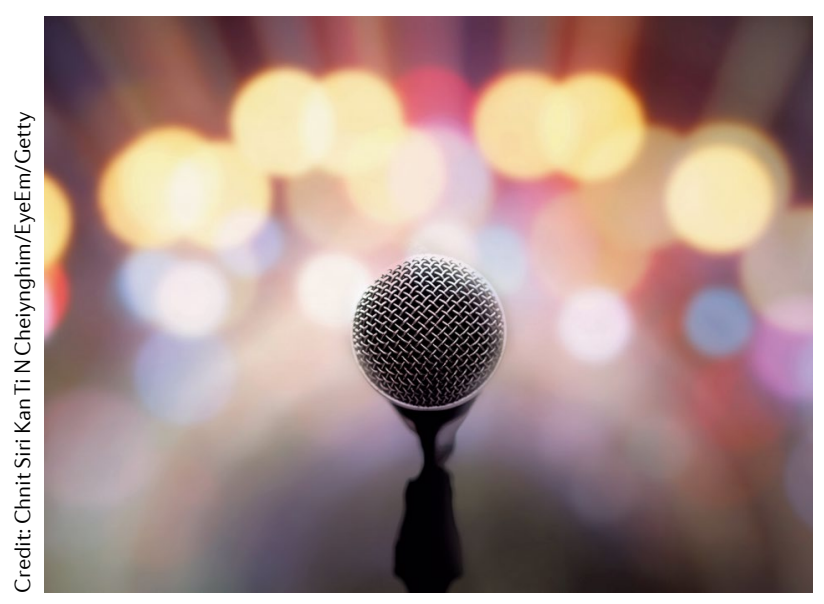

arrangements of genes across multiple plasmids. To test for successful gas vesicle generation, the authors transfected the plasmids into human embryonic kidney (HEK) 293 cells, enriching for the resultant gas vesicles by taking the most buoyant fraction of the cell lysate and imaging the lysates by transmission electron microscopy. A nine-gene system re-coded from Bacillus megaterium and expressed from three plasmids at 1-2 copies per gene resulted in robust gas vesicle generation. Similar results were achieved in Chinese hamster ovary cells. For further follow-up studies, the authors selected and expanded a HEK293 subclone that contained an inducible form of the ARG constructs and robustly formed gas vesicles following doxycycline treatment.

The investigators tested how the gas vesicles could be detected using ultrasound, rather than being directly visualized by electron microscopy. Due to the background sound scattering properties of cells, standard ultrasound was suboptimal for distinguishing cells with from those without gas vesicles. However, a dynamic modulation pulse sequence of ultrasound resulted in rupturing ('collapse') of the gas vesicles, which facilitated their detection based on contrast changes before and after collapse. Although gas vesicles are destroyed by this ultrasound pattern, they could be redetected after the ARGs were re-expressed over the following 3 days.

Importantly, the method showed applicability in vivo: when the HEK293 cells formed xenografts in mice, systemic administration of doxycycline resulted in an ultrasound signal at the periphery of the tumours. Given the increased vascularization (and hence doxycycline delivery) at the periphery, this distribution was to be expected and could be recapitulated by histological analysis. However, this pattern was not discernible by in vivo imaging of a fluorescent reporter also expressed by the constructs, due to poor light penetration.

One potential concern of this reporter system is that the generation of gas vesicles could be toxic to cells in vitro or in vivo. Although further work is required to fully address this issue, the authors found only minor effects of ARG expression on cell viability, and they note that the gas vesicles represent only $0.0027 \%$ of the cell volume.

Overall, the most notable achievement of this study expressing a complex set of interdependent bacterial genes in mammalian cells - is also likely to be its greatest challenge for wider adoption. The authors suggest several future redesign approaches for streamlining the current threeplasmid system into fewer plasmids and reducing the overall payload size. They draw parallels with fluorescent protein expression constructs, which underwent several years of development to optimize expression and optical properties. The aim is that ultrasound-based reporter systems could be widely delivered to a variety of mammalian experimental systems in vitro and in vivo, to provide robust reporter expression without the need to select individual high-expressing subclones.

Further avenues for exploration include rewiring the system to report on endogenous genes of interest and adapting the dynamic properties to allow more-frequent detection, either by devising a non-collapsing detection procedure or increasing the rate of gas vesicle re-expression after collapse.

Darren J. Burgess

ORIGINAL ARTICLE Farhadi, A. et al. Ultrasound imaging of gene expression in mammalian cells. Science 365, 1469-1475 (2019) 